\title{
ANÁLISE DE UM EPISÓDIO DE NUVEM FUNIL EM SANTA MARIA/RS NO DIA 4 DE MARÇO DE 2016.
}

\author{
Amanda ComassettoIensse ${ }^{(a)}$, Cássio Arthur Wollmann ${ }^{(b)}$, Ricardo Stedile Neto $^{(\mathrm{c})}$ \\ (a) Programa de Pós-Graduação em Geografia, Universidade Federal de Santa Maria, comassettoamanda@gmail.com \\ (b) Departamento de Geociências, Universidade Federal de Santa Maria, cassio_geo@ yahoo.com.br

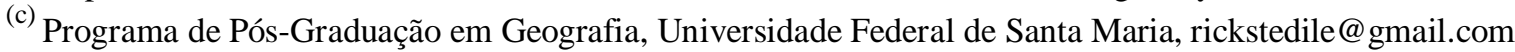

Eixo: Climatologia em diferentes níveis escalares: mudanças e variabilidades.

Resumo:

No Brasil, sobretudo no centro-sul, os eventos extremos como tornados são registrados frequentemente pela população. Além dos tornados, também são registradas as nuvens funil que tem a mesma gênese do tornado, mas não toca o solo. $\mathrm{O}$ objetivo desse trabalho foi de analisar as condições atmosféricas que resultaram na ocorrência de uma nuvem funil em Santa Maria/RS no dia 04 de março de 2016. Os procedimentos metodológicos baseados em cartas sinóticas e dados da estação meteorológica de Santa Maria, mostraram-se propícios para a formação de distúrbios na atmosfera resultando na nuvem funil.

Palavras-chaves: Nuvem funil, Santa Maria, Evento Tornádico.

\section{Introdução}

Os eventos climáticos extremos não são raros no Rio Grande do Sul, pois o estado constantemente é atingido por altas e baixas pressões causando instabilidades e, dessa forma proporcionando a formação de eventos extremos. Dentre os eventos climáticos extremos, encontram-se os tornados, trombas d’água e nuvens funil. Publicações tratando desse assunto já foram produzidas por alguns pesquisadores no Brasil, Lima (1982), Silva Dias e Grammelsbacher (1991), Massambaniet al. (1992), Nechet (2002), Marcelino (2004), Reckziegel (2007), Rodrigues(2011) e Candido (2012).

A dinâmica da atmosfera faz parte das manifestações da natureza e ocorrerá com ou sem a presença do homem, e suas consequências podem variar. A preocupação maior a cerca desses fenômenos naturais é quando resulta em vítimas fatais e com o aumento da população seja urbana ou rural, tais eventos acabam por serem registrados mais frequentemente.

No Brasil, sobretudo no centro-sul, os tornados e as nuvens funil principalmente são registrados frequentemente pela população que fica fascinada e amedrontada ao mesmo tempo. Com o avanço da tecnologia esses registros ficaram mais fáceis, sendo comum a população possuir uma câmera ou um 
XVII Simpósio Brasileiro

de Geografia Física Aplicada

I Congresso Nacional

de Geografia Física
OS DESAFIOS DA GEOGRAFIA FÍSICA NA FRONTEIRA DO CONHECIMENTO

Instituto de Geociências - Unicamp

Campinas - SP

28 de Junho à 02 de Julho de 2017

celular para fazer o registro. Ao mesmo tempo em que os registros foram facilitados pela tecnologia ainda é difícil construir um banco de dados fiel principalmente às nuvens funil.

São muitas as definições sobre tornados e nuvens funil encontradas na literatura, mas é consenso que o tornado é um fenômeno forte e perigoso, caracterizado por uma coluna de ar ascendente em contato com a superfície e gira violentamente a vários metros por segundo. A nuvem funil por sua vez tem a mesma gênese do tornado, mas não toca o solo. Segundo Nechet (2002) nuvem funil é uma coluna de ar, que gira pendente de uma nuvem cumulonimbus, criando movimentos verticais de baixo para cima, mas que não toca o solo ou a superfície líquida.

Este trabalho tem o objetivo de analisar as condições atmosféricas que proporcionaram a formação de uma nuvem funil no município de Santa Maria, no Rio Grande do Sul no dia 04 de março de 2016.

\section{Fundamentação teórica}

Os tornados derivam de nuvens chamadas Cumulonimbos $(\mathrm{Cb})$ e esses eventos normalmente são decorrentes de nuvens de elevado desenvolvimento vertical e intensa turbulência interna (FUJITA; PEARSON, 1973). Apesar de estar próxima a superfície terrestre, pode chegar a níveis muito altos na atmosfera, até o topo da troposfera. Essas nuvens acumulam muita energia interna, às vezes causam ventos fortes, precipitação, relâmpagos e granizo aparecendo isoladas. (WAKIMOTO; WILSON, 1989). É de suma importância reconhecer que uma $\mathrm{Cb}$ formou um tornado de fato só quando esse toca o solo, do contrário o evento é uma nuvem funil.

Nuvens funil não são raras no território brasileiro sendo observada em diversos estados e em todas as estações do ano. As nuvens funil tem a mesma gênese de um tornado, porém não há umidade e vorticidade o suficiente dentro da nuvem para o desenvolvimento do funil até tocar o solo. Allaby (2004) coloca que nem toda nuvem de tempestade apresenta tornados, mas qualquer formação de origem convectiva ou frontal que apresente condições propícias ao desenvolvimento de tornados trombas d'água ou nuvem funil é chamada de tempestade tornádica.

Em 05 de dezembro de 1975, Santa Maria/RS registrou o primeiro evento tornádico em território brasileiro. Por se tratar de um evento, na época, descrito como raro, o episódio foi analisado por José Soares Lima, Meteorologista Chefe do centro de meteorologia de Porto Alegre. Lima (1982) colocou que é de vital importância conhecer a causa da natureza do tempo severo. O registro da nuvem funil de 1975 foi vista pelo observador de serviço da estação meteorológica de Santa Maria e foi fotografado diversas vezes por um militar especialista em fotografia. 


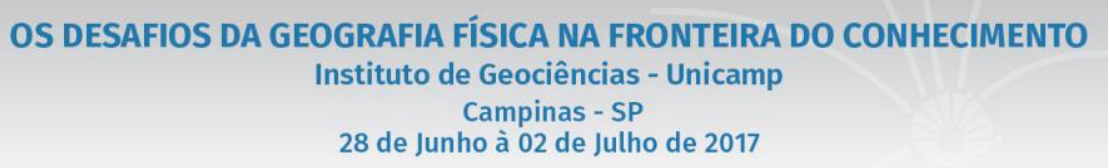

A figura 01 mostra a foto da nuvem funil registrada no dia 05 de dezembro de 1975 no setor sudoeste do aeroporto, cerca de $5 \mathrm{~km}$ de distância.

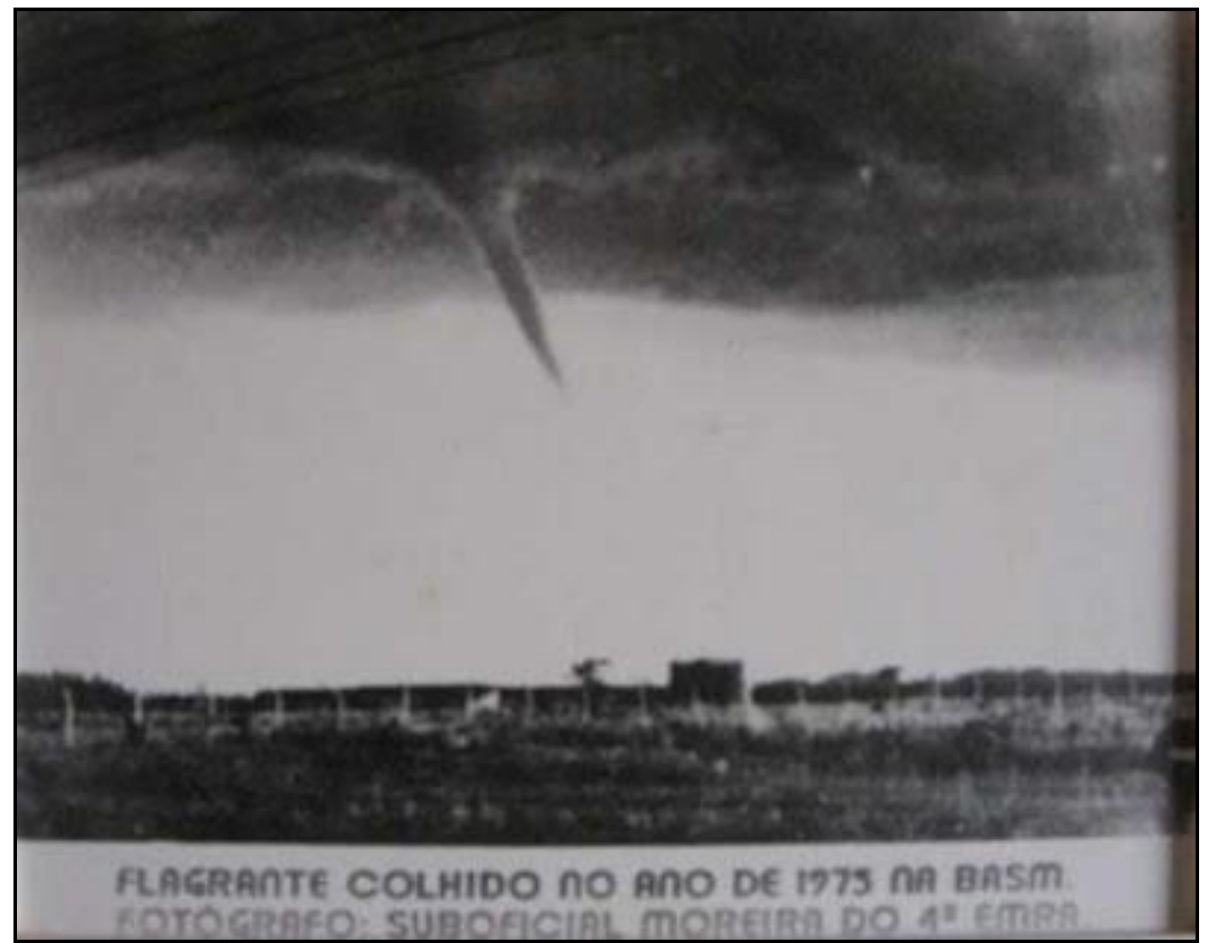

Figura 01 - foto da nuvem funil registrada no dia 05 de dezembro de 1975 na Base Aérea de Santa Maria. Fonte: Suboficial Moacir Moreira

\subsection{Localização da área de estudo}

O município de Santa Maria (Figura 02) está localizado no centro do estado do Rio Grande do Sul entre as coordenadas $29^{\circ} 41^{\prime} 02^{\prime \prime}$ Sul e $53^{\circ} 48^{\prime} 25^{\prime \prime}$ Oeste e está na depressão central sul-rio-grandense. A região pertence à Bacia do Paraná, com Cobertura Sedimentar Gonduânica, pertencente à Formação Botucatu, constituída por arenito, sendo produto de vulcanismo ácido e básico (KAUL, 1990). 


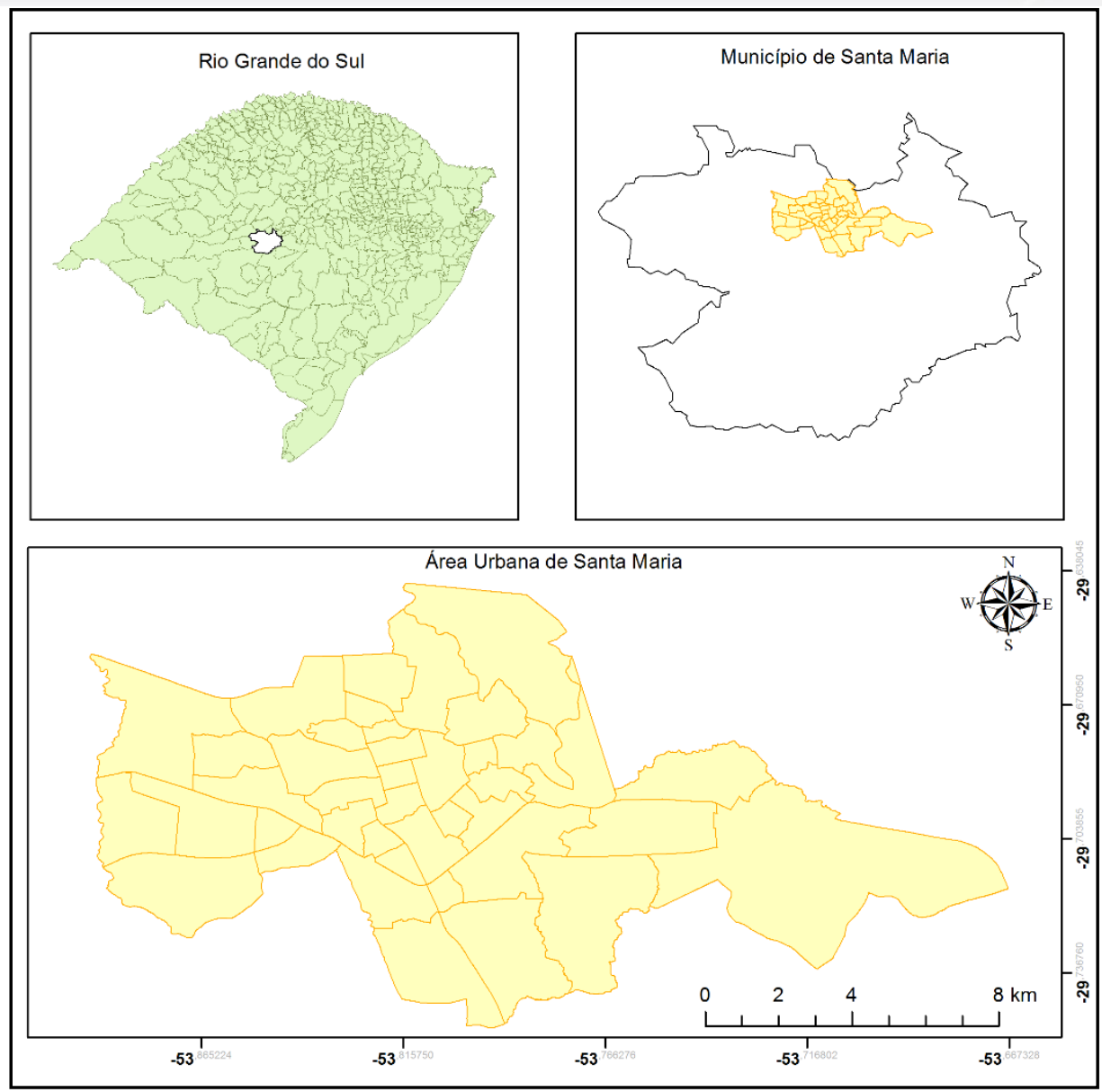

Figura 02 - Localização da área de estudo.

Org.: Amanda ComassettoIensse.

\section{Procedimentos metodológicos}

Os procedimentos metodológicos desse artigo foram divididos em etapas. Analisou-se as cartas sinóticas das $00 \mathrm{~h}$ e das $12 \mathrm{~h}$ dos dias 03 e 04 de março de 2016 afim de identificar os sistemas atmosféricos envolvidos na gênese da nuvem funil. Também foram utilizadas imagens de satélite para corroborar os dados da estação meteorológica de Santa Maria. Os dados utilizados da estação foram de temperatura, umidade relativa, pressão, velocidade dos ventos e nebulosidade. Além dos dados meteorológicos foi feito um trabalho de campo no setor da cidade onde o evento ocorreu para averiguar se houve estragos.

\section{$4 \quad$ Resultados e discussões}

No dia 03 de março de 2016, identificou-se um cavado sobre o Rio Grande do Sul na carta sinótica da 00h e na carta sinótica das $12 \mathrm{~h}$. No dia 04 de março de 2016 Santa Maria estava sobre a influência de um 


$\begin{aligned} & \text { XVII Simpósio Brasileiro } \\ & \text { de Geografia Fisica Aplicada }\end{aligned}$
$\begin{aligned} & \text { I Congresso Nacional } \\ & \text { de Geografia Física }\end{aligned}$

centro de baixa pressão que se originou no cavado que atuava sobre o Rio Grande do Sul no dia anterior, além de um sistema frontal na altura de Buenos Aires na Argentina.

As cartas sinóticas dos dias 03 e 04 de março podem ser observadas na figura 3.

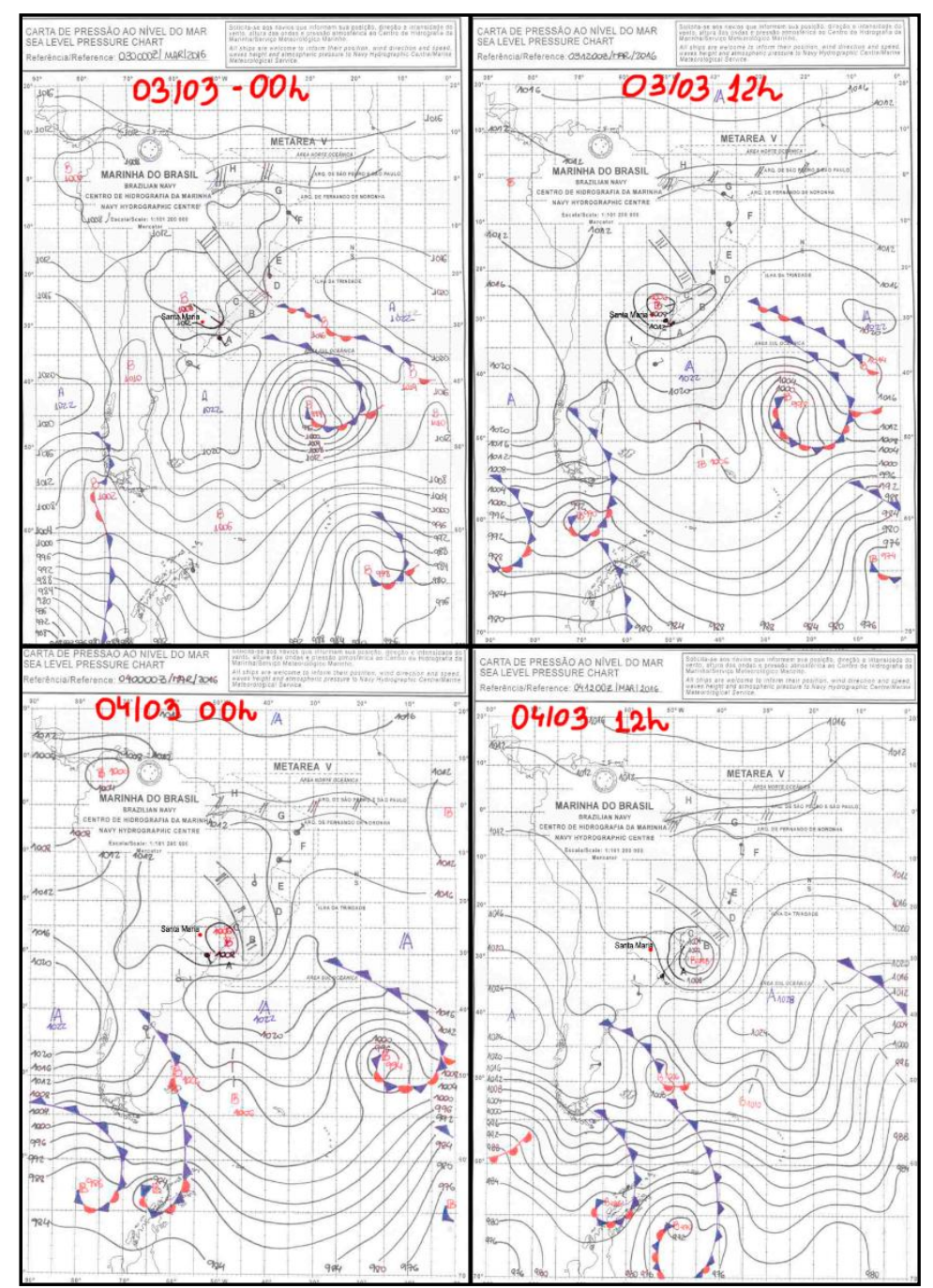

Figura 3 - Cartas sinóticas dos dias 03 e 04 de março de 2016 Fonte: Marinha do Brasil

A imagem de satélite do dia anterior ao evento mostra o Rio Grande do Sul com muita nebulosidade devido ao cavado presente na carta sinótica. Por outro lado, no dia do evento a região central do Rio Grande do Sul, onde encontra-se Santa Maria, aparece com nebulosidade devido ao seu domínio préfrontal.

A figura 4 mostra as imagens de satélite dos dias 03 e 04 de março de 2016. 

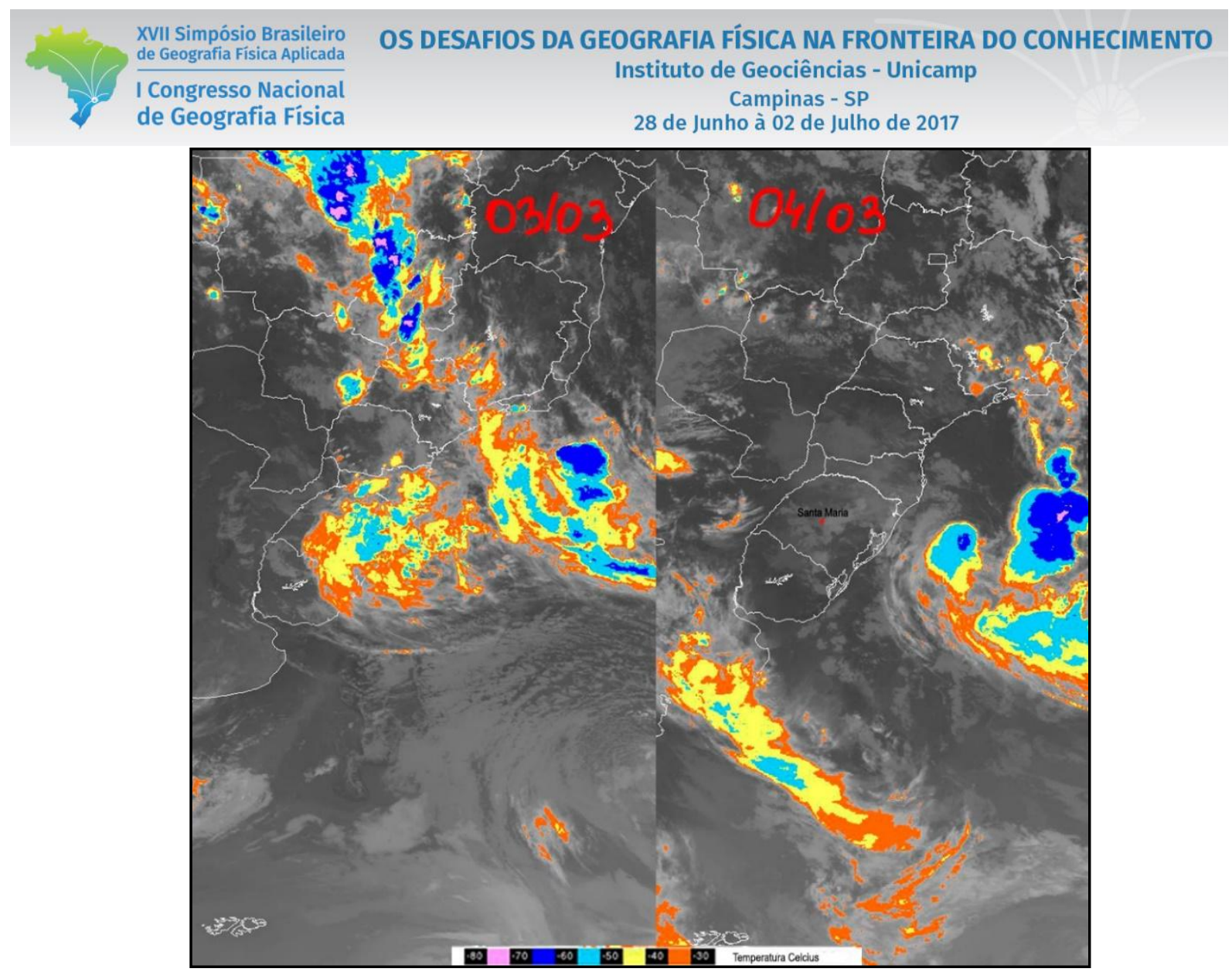

Figura 4- Imagem de satélite GOES-13 T_realçada

Fonte: acervo CPTECINPE

Sartori (1993) definiu a sucessão dos tipos de tempo através de fases evolutivas sendo que no dia 04 de março, Santa Maria encontrava-se em domínio pré-frontal com pressão atmosférica em declínio e ventos do quadrante norte. Em escala regional o vento norte está associado a fortes gradientes barométricos e em escala local pelo condicionamento do rebordo do planalto o vento norte intensifica-se ainda mais.

Os dados da estação meteorológica de Santa Maria dos dias 03 e 04 de março podem ser observados na tabela 1 .

Tabela I - Dados da estação meteorológica de Santa Maria dos dias 03 e 04 de março de 2016

\begin{tabular}{|c|c|c|c|c|c|c|}
\hline Data & Hora & $\begin{array}{c}\text { Pressão } \\
\text { Atmosférica } \\
(\text { mbar })\end{array}$ & $\begin{array}{c}\text { Umidade } \\
\text { Relativa } \\
(\%)\end{array}$ & Nebulosidade & $\begin{array}{c}\text { Temperatura } \\
\left(\mathbf{C}^{\mathbf{0}}\right)\end{array}$ & $\begin{array}{c}\text { Vel. } \\
\text { dos } \\
\text { ventos } \\
(\mathbf{m} / \mathbf{s})\end{array}$ \\
\hline $\mathbf{0 3 / 0 3 / 2 0 1 6}$ & $\mathbf{0 0}$ & $\mathbf{1 0 0 0 . 8}$ & $\mathbf{9 2}$ & $\mathbf{1 0}$ & $\mathbf{2 0 . 4}$ & $\mathbf{3 . 3}$ \\
\hline $\mathbf{0 3 / 0 3 / 2 0 1 6}$ & 12 & 998.2 & 90 & 10 & 18.8 & $\mathbf{5 . 8}$ \\
\hline $\mathbf{0 3 / 0 3 / 2 0 1 6}$ & 18 & $\mathbf{9 9 7 . 9}$ & $\mathbf{9 5}$ & $\mathbf{1 0}$ & $\mathbf{1 9 . 8}$ & $\mathbf{8 . 1}$ \\
\hline $\mathbf{0 4 / 0 3 / 2 0 1 6}$ & $\mathbf{0 0}$ & $\mathbf{9 9 9 . 8}$ & $\mathbf{8 7}$ & $\mathbf{1 0}$ & $\mathbf{2 0 . 2}$ & $\mathbf{1 . 3}$ \\
\hline $\mathbf{0 4 / 0 3 / 2 0 1 6}$ & 12 & $\mathbf{1 0 0 1 . 2}$ & $\mathbf{9 2}$ & $\mathbf{1 0}$ & $\mathbf{2 0 . 2}$ & $\mathbf{1 . 3}$ \\
\hline $\mathbf{0 4 / 0 3 / 2 0 1 6}$ & 18 & $\mathbf{9 9 9 . 5}$ & $\mathbf{6 4}$ & $\mathbf{7}$ & $\mathbf{2 8 . 2}$ & $\mathbf{1 . 6}$ \\
\hline
\end{tabular}




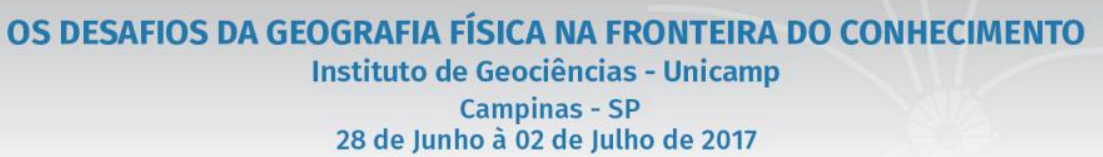

28 de Junho à 02 de Julho de 2017

De acordo com a tabela 1, nota-se oscilação barométrica presente na atmosfera de Santa Maria, alta umidade relativa do ar e céu totalmente encoberto. A temperatura e velocidade dos ventos também oscilaram ao longo dos dois dias. Os dados meteorológicos coletados do dia anterior ao evento e do dia do evento, mostram-se propícios para a formação de distúrbios na atmosfera, os quais resultaram na formação de uma nuvem funil no dia 04 de março de 2016.

O registro da nuvem funil em Santa Maria, foi feita no bairro Tancredo Neves, sudoeste da cidade e foi fotografada e filmada por muitos moradores. A figura 5 mostra imagens feitas da nuvem funil a partir de 3 pontos distintos do bairro.

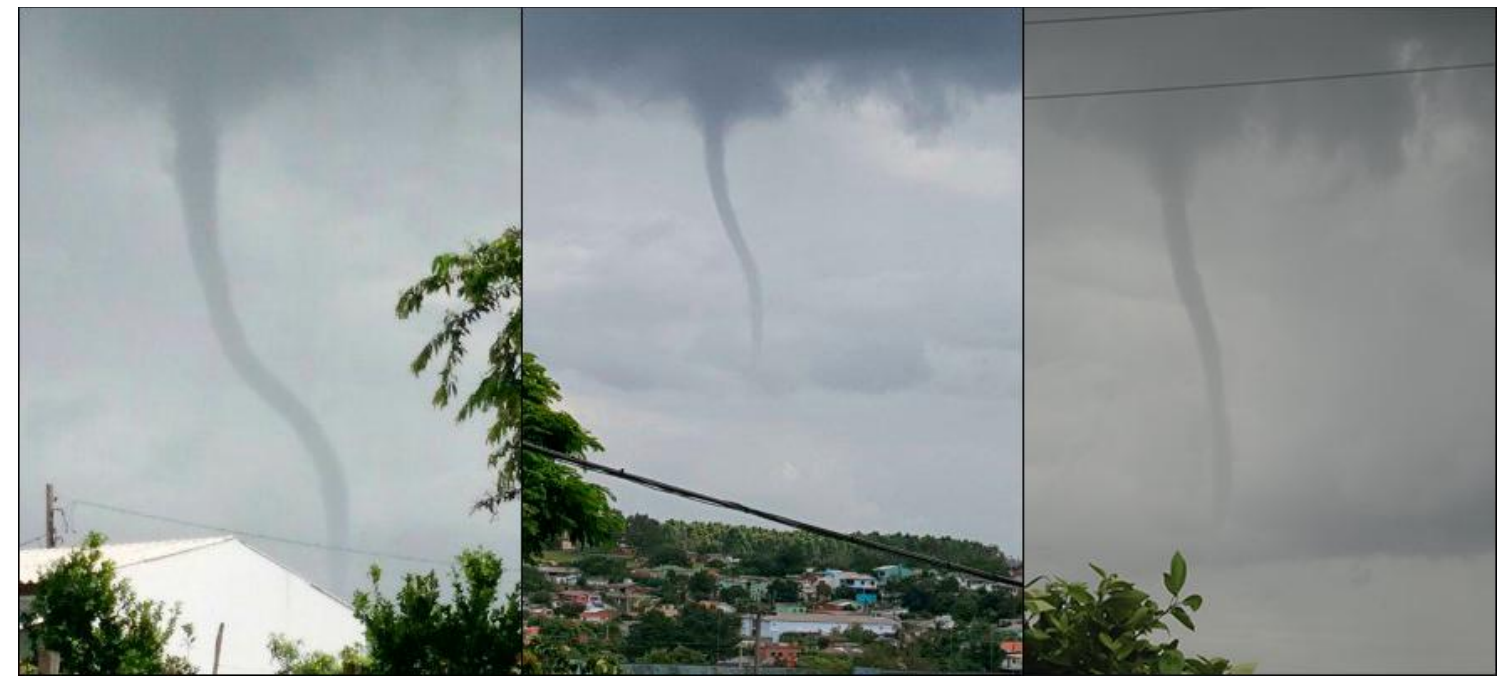

Figura 5 - Imagens da nuvem funil no dia 04/03/2016 em Santa Maria/RS Fonte: Juliano Costa, Diego Souza e Ricardo StedileNeto

O registro da nuvem funil foi feito entre $15 \mathrm{~h}$ e $16 \mathrm{~h}$ e segundo os moradores que presenciaram o fenômeno, a nuvem funil permaneceu no céu por cerca de 15 minutos até se dissipar. A partir do trabalho de campo feito no dia posterior ao evento, constatou-se que não houve nenhum tipo de dano no local da ocorrência. Alguns moradores que residem bem próximos ao local do evento foram entrevistados a respeito do ocorrido.

As pessoas que avistaram a nuvem funil a cerca de $5 \mathrm{~km}$, não sentiram nenhum efeito sobre o ocorrido e muitos moradores bem próximos ao local do evento, afirmaram que só ficaram sabendo após ouvir na rádio.

\section{Conclusões}

A nuvem funil ocorrida no dia 04 de março em Santa Maria, não é um evento raro na região. E eventos como esse tem sido registrado frequentemente graças a tecnologia que possuímos hoje, como os celulares 
e as câmeras digitais. Os dados de carta sinótica e imagens de satélite foram cruciais para corroborar os dados da estação meteorológica e também das imagens capturadas pela população.

Durante a análise do episódio de nuvem funil, constatou-se também a necessidade de mais pesquisa e estudos de caso sobre esse tipo de evento que não é raro e merece atenção.

\section{Referencias}

ALLABY, M. Tornadoes.Facts on File: New York, 2004.

CANDIDO, D. H. tornados e trombas d'água no Brasil: Desenvolvimento de um modelo e proposta de escala de avaliação de danos. 2012. 230 f. Tese (Doutorado em Geociências) - Universidade Estadual de Campinas. 2012.

FUJITA, T.T.; PEARSON, A.D.: Results of FPP classification of 1971;1972 tornadoes. Preprints, 8th Conf on Severe Local Storms, Denver, Amer. Meteor.Soc. 1973.

KAUL, P. F. T. Geologia. In: IBGE. Geografia do Brasil: região sul. Rio de Janeiro, 1990. p. 29- 54.

LIMA, J.S. Ocorrência de uma Nuvem Funil em Santa Maria-RS, In: Anais do Segundo Congresso Brasileiro de Meteorologia. Pelotas, RS: 1982.

NECHET, D. Ocorrência de tornados no Brasil. Boletim da Sociedade Brasileira de Meteorologia, v. 26, n. 2, p. 29-39, dez. 2002.

RODRIGUES, M.L.G., et al... CLIMATOLOGIA DE FRENTES FRIAS NO LITORAL DE SANTA CATARINA. Revista Brasileira de Geofísica: 135-151. 2004.

SARTORI, M. G. B. A circulação atmosférica regional e os principais tipos de sucessão do tempo no inverno do Rio Grande do Sul, Brasil. Ciência e Natura, n. 15, p. 69-93, 1993.

SILVA DIAS, M.A.F. e GRAMMELSBACHER, E. A. A possível ocorrência de tornado em São Paulo no dia 26 de abril de 1991: um estudo de caso. Revista Brasileira de Meteorologia, v. 6, n. 2, p. 513-522, 1991.

WAKIMOTO, R. M.; WILSON, J. W. Nonsupercell Tornadoes.Monthly Weather Review: Vol. 117, No. 6, pp. 1113 1140. - Review: 1113-1140. 1989. 